\title{
Una iglesia de campo Parte I
}




\section{Daniel Plenc}

Villa Libertador San Martín

Entre Ríos, Argentina 


\section{RESUMEN}

"Una iglesia de campo - Parte I" - El autor hace una visita a una iglesia de años de trayectoria fiel. Es la iglesia adventista de Colonia Pintos Viana, Guichón, Uruguay. Viejas memorias reviven en fotos, ambiente y sobre todo en familias que por décadas han venido disfrutando del gozo en el mensaje de la bienaventurada esperanza. Las páginas de los libros de iglesia atestiguan los 86 años de su trayectoria feliz. Cada acto de la celebración de su fundación mostró la fe bíblica arraigada en esos creyentes que viven con corazones sinceros para Dios.

\section{SUMMARY}

"A Country Church - Part I" - The author visits a church that has had years of faithful history. It is the Adventist Church of Colonia Pintos Viana, Guichon, in Uruguay. Old memories were revived in photos, the environment, and over all in the families that for decades have enjoyed the happiness of the message of the blessed hope. The pages of the church books also witness the 86 years of its happy history. Each act of celebration from its foundation has showed the biblical faith rooted in those believers that live with sincere hearts for God. 


\section{UNA IGLESIA DE CAMPO \\ Parte I}

La mañana del domingo 28 de enero de 2007 se presentaba espléndida. Ese día ocurriría una nueva edición de la histórica reunión de la familia Cayrus, junto al arroyo Santana en la Colonia Pintos Viana, a poco más de diez kilómetros de Guichón. El paisaje campestre lucía verde, profundo, infinito. El silencio, sólo interrumpido por el canto de las cotorras y otras aves desde las ramas de los eucaliptos, invitaba a la paz del espíritu. Una brisa incesante ofrecía su alivio en ese día de verano. En medio de ese paraje bucólico se erguía la capilla, a cuyos archivos había decidido acudir en procura de registros que permitieran reconstruir la historia de la iglesia.

Me senté en uno de los bancos de la escuela de otro tiempo, observé las fotografías, tomé nota de las inscripciones y supe que aquel era un lugar especial. Una fotografía de 1924 mostraba a un grupo de alumnos y de miembros de la iglesia. ${ }^{1}$ Del mismo año es el retrato de

1Son ellos: Esli, Inés y Benoní Cayrus; Nicolás Mazur; Sarita Racovsky; Juan Oleynick; Constancia de Cayrus; David Plenc, con su hijito Walter en brazos; Pedro Cayrus, los pastores Ner Soto Garrido y A. R. Sherman; Juan Racovsky; Miguel Dreher; Iván Racovsky. En la última fila Margarita Cayrus; Elena Cayrus de Plenc con su hijito Elbio; Emilia y Emilio Cayrus; Enrique Cayrus; la Sra. Racovsky; Cristina de Dreher; María Fontana de Cayrus; Lelia, Alda y Paulina Cayrus. 
otras personas, entre ellas algunas mujeres vestidas de luto, tal vez por el fallecimiento de Juan Elías Cayrus. ${ }^{2}$ Otra fotografía expone a tres líderes de la iglesia en 1926, los pastores Soto y Krieghoff, elegantemente sentados en un carro liviano y en un caballo, el hermano Juan Emilio Cayrus.

\section{La Escuela Sabática}

La iglesia conserva libros de registros desde sus inicios en 1923. El humilde grupo de creyentes, de unas pocas familias rurales, había dado origen a una Escuela Sabática, luego a una iglesia y a una escuela confesional. $^{3}$ En las manos de la Providencia esas instituciones habrían de afirmar la fe de los creyentes, algunos de los cuales han servido ampliamente a la causa del adventismo sudamericano. Serían también agentes en la evangelización de esa amplia zona rural del Departamento Paysandú, en la República Oriental del Uruguay.

La Escuela Sabática se organiza el 6 de noviembre de 1923, por sugerencia del pastor Carlos E. Krieghoff, entonces presidente de la Misión Uruguaya de la Iglesia

${ }^{2}$ La fotografía muestra a las siguientes personas: Juan Emilio Cayrus, Racovsky, David Plenc, Miguel Dreher, Carlos Krieghoff, Eumen Racovsky, Constancia C. de Cayrus y Elena Cayrus de Plenc. En una segunda fila: Pablo Enrique Cayrus, Iván Racovsky, Margarita Cayrus, Cristina de Dreher, Sra. Racovsky, Emilia Dreher de Cayrus, Paulina y Alina Cayrus.

${ }^{3}$ En la estancia Santa Isabel había funcionado una escuela pública a la cual concurrían los niños de la colonia hasta el tercer grado. En el futuro los adventistas sintieron la necesidad de contar con una escuela de iglesia. 
Adventista del Séptimo Día. La propuesta fue aceptada con gusto por los 30 asistentes a la reunión. Una comisión de nombramientos integrada por los hermanos Eumen Racovsky, Miguel Dreher y Juan Emilio Cayrus fue la encargada de presentar a la asamblea una propuesta de sus oficiales y maestros. ${ }^{4}$ Se autorizó también el funcionamiento de una clase de menores. ${ }^{5}$ En los años siguientes se agregarían nuevas clases para los niños más pequeños y también una clase para jóvenes. Antes del cierre de aquella primera reunión organizadora, se dieron instrucciones oportunas acerca del propósito redentor y misionero de la Escuela Sabática.

Así fue que la Escuela Sabática dio comienzo formal el sábado 10 de noviembre de 1923, con un himno y la oración del director. El misionero trimestral que hablaba acerca de la obra adventista en la Unión Incaica fue leído por Paulina Cayrus. Diez adultos y cuatro menores se inscriben en las clases. El repaso de la lección fue dirigido por David Plenc y la lección del día, que trataba acerca del amor, fue conducida por la maestra recientemente designada, Elena Cayrus. ${ }^{6}$ Se concluye

${ }^{4}$ Los oficiales de la Escuela Sabática designados para lo que restaba de 1923 y el primer semestre de 1924 fueron los siguientes. Director: David Plenc, subdirector: Carlos Racovsky, secretario: Juan Emilio Cayrus, subsecretaria: Emilia Dreher, maestro para los de habla rusa: Antonio Mazur, maestro para los de habla castellana: Elena Cayrus.

${ }^{5} \mathrm{La}$ organización de la clase de menores se concretó el 24 de noviembre de 1923 con el nombramiento de Paulina Cayrus como maestra. Paulina Cayrus de Barboza, de 102 años, es el miembro más antiguo de la familia Cayrus al momento de escribirse este trabajo.

${ }^{6}$ Elena María Cayrus, nacida en Italia (1899-1997) y David Plenc (1899-1992) habrían de contraer matrimonio el 6 de marzo de 1924, en Guichón. Se mantuvieron fieles al mensaje adventista por muchos años 
con otro himno y la oración de la maestra. En el futuro se habrían de tomar decisiones adicionales. Se pensó que lo mejor era celebrar la Escuela Sabática en diferentes lugares, en casa de la familia Cayrus en Guichón, en el casco de la antigua estancia Santa Isabel que Juan Elías Cayrus había adquirido, en casa de Eumen Racovsky o de Miguel Dreher, entre otros. Las ofrendas se recogían primero en forma trimestral y luego semanalmente. El horario de inicio, necesariamente flexible, también fue cambiando.

Hubo modificaciones y diferencias de criterio, pero las reuniones de la Escuela Sabática no cesaron. Aquellos encuentros incluían alabanzas, oraciones, estudio de la Biblia e instrucción misionera. Los himnos formaban una parte significativa de la sencilla liturgia. Puede leerse en los cuadernillos de recuerdos de la familia Cayrus que las primeras reuniones religiosas habían consistido a menudo solamente en la entonación de himnos, en español o en ruso. El libro de actas consigna los títulos de unos 60 himnos diferentes que se entonaron durante el primer año de reuniones. La participación del reducido grupo era necesariamente activa. Apellidos como Cayrus, Dreher, Plenc, Racovsky $\mathrm{y}$ otros se repiten en los informes. Estos primeros miembros, algunos de ellos jóvenes no bautizados, leían los informes misioneros y enseñaban las Escrituras.

Existían secciones fijas en la Escuela Sabática que diferían un poco de las actuales. El informe misionero mundial eran infaltable, lo mismo que la tabla compara-

tanto en la zona rural de Guichón y Pandule como en la ciudad de San José de Mayo. 
tiva con sus blancos de ofrendas misioneras. Las ofrendas del décimo tercer sábado y de cumpleaños estaban destinadas al campo mundial. Se hacía un repaso de la lección de la semana anterior y se desarrollaba la del día. La persona encargada de la secretaría debía leer el informe del sábado anterior y someterlo a votación. Generalmente era aprobado, pero había posibilidades de hacer observaciones y enmiendas. El décimo tercer sábado era un día especial, que ofrecía a los niños y jóvenes oportunidad de participar. Se realizaban diálogos, se presentaban himnos especiales y se recitaban poesías. Pareciera que se aprovechaba esa circunstancia para realizar una recapitulación de la lección de todo el trimestre.

Los asistentes, entre miembros y visitas, no eran muchos. Al comienzo se daban cita entre doce y veintiséis miembros, al igual que un número muy variable de visitantes. El clima y la visita de algún pastor solían influir en la afluencia de personas al lugar de reunión. Sin embargo, el objetivo misionero de la Escuela Sabática era claro y el crecimiento era inevitable. En mayo de 1924 ya había personas deseosas de ser bautizadas en el arroyo cercano.

\section{Se organiza la Iglesia}

No fueron pocos, de cerca y de lejos, los que hicieron su parte para que la Escuela Sabática fuera transformándose en una iglesia. Cuando llegaban visitantes, seguramente tenían participación en los cultos. Los obreros adventistas que venían de Paysandú o de Montevideo dirigían cultos, la Cena del Señor y los bautismos, además de presidir los nombramientos de los nuevos oficiales. La celebración de la Santa Cena daba 
ocasión para la reflexión bíblica y para que los hermanos compartieran su testimonio. Con cierta regularidad se acercaban otros miembros de la Misión, como su tesorero, el pastor Ner Soto Garrido o los directores de departamentos. Los pastores del distrito de Paysandú, como A. R. Sherman, eran visitantes frecuentes. Los nombres de muchos de estos misioneros han permanecido en el recuerdo de los creyentes. Los libros muestran nombres como los de José Replogle, J. M. Howell, Marcelo Pidoux, Humberto Cairus, Walter Brown, Adolfo Lista, Héctor Pereyra, Jorge Iuorno, Santiago Bernhardt, Alfredo Aeschlimann, Daniel Nestares, Alfredo Bellido de la Fuente, Carlos Polischuk y otros. Los presidentes de la Misión Uruguaya mencionados en esos años fueron C. E. Krieghoff, Pedro M. Brouchy, Enrique Westhpal, Niels Wensell y Juan Riffel.

El tiempo había llegado para que los hermanos discutieran la posibilidad de organizar una iglesia. Se nombraron los primeros oficiales, según el siguiente listado. Anciano y director misionero: Miguel Dreher, diácono: Eumen Racovsky, diaconisa: Constancia Davit de Cayrus, secretario: Juan Emilio Cayrus, tesorero: Carlos Racovsky, secretaria misionera: Elena Cayrus de Plenc, director de jóvenes: David Plenc. ${ }^{7}$ Se da continuidad a una clase de Escuela Sabática en ruso y otra en castellano. Carlos Racovsky se transforma en maestro para la clase en ruso y Pedro Cayrus para la clase en castellano.

El libro de actas de la iglesia dice textualmente:

${ }^{7}$ Se nombra un comité para la iglesia integrado por Miguel Dreher, Eumen Racovsky, Carlos Racovsky, Constancia D. de Cayrus, Elena Cayrus de Plenc y Juan Emilio Cayrus. 
“El día 11 de mayo de 1924 se reunieron en la casa de la familia Cayrus los hermanos [...] que viven en la Colonia Pintos Viana, cerca de Guichón, Departamento Paysandú y los pastores A. R. Sherman y C. E. Krieghoff, el último en su calidad de superintendente de la Misión Uruguaya de los Adventistas del Séptimo Día. Los hermanos acordaron unánimemente organizarse en una iglesia, la cual llevaría el nombre de Iglesia Adventista de Guichón". En ese día se celebraron las "ordenanzas del Señor" con la participación de todos. Escribió el secretario: "El Señor se hizo sentir muy cerca de nosotros".

El núcleo primitivo de la iglesia recién organizada estuvo integrado por los hermanos Miguel Dreher, Ernestina de Dreher, David Plenc, Elena Cayrus de Plenc, Juan Emilio Cayrus y Pedro Cayrus. Los hermanos que pertenecían a la iglesia de la Misión podían elegir solicitar su carta de traslado a la nueva iglesia. Además se recibió a los hermanos bautizados el día anterior: María Fontana de Cayrus, Constancia Davit de Cayrus, Paulina Cayrus, Pablo Enrique Cayrus, Margarita Cayrus, Alina Berta Cayrus, Emilia Dreher y Juan Racovsky. ${ }^{8}$ La iglesia aceptó también a los matrimonios rusos Eumen y Matilde Racovsky, Carlos y Natalia Racovsky, que habían sido bautizados con anterioridad.

${ }^{8}$ María Fontana de Cayrus, nació en Italia el 13 de octubre de 1844, y falleció en Guichón, Uruguay, el 5 de mayo de 1939. Constancia Davit de Cayrus, nacida en Italia el 12 de enero de 1878, falleció el 13 de enero de 1975. Juan Elías Cayrus, nacido también en Italia el 10 de mayo de 1877, falleció el 14 de julio de 1923, sin ver el surgimiento de la obra organizada. 


\section{Limitaciones y desarrollo}

La membresía de la iglesia creció con los años, con nuevos bautismos y cartas de traslado. ${ }^{9}$ Los niños crecían y se comprometían con el Señor y también nuevas personas se acercaban a la iglesia deseando pertenecer a ella por medio del bautismo o la profesión de fe. ${ }^{10}$ También hubo hermanos que dejaron la iglesia y se alejaron de ella por razones doctrinales o particulares. ${ }^{11}$

${ }^{9}$ Algunas personas recibidas por carta de traslado fueron: Jesús Godoy, desde Paysandú (1937), Alda Cayrus de Geisse y Günther Geisse, desde la Misión Boliviana (1937), Nancy Malán de Dreher, desde Nueva Helvecia, Alba M. de Bergalli, desde Paysandú (1947), Adela Turosky, de la iglesia de la Misión y Teodoro Kosiak, de Paysandú (1953).

${ }^{10}$ Una lista parcial de personas bautizadas o recibidas es la siguiente: Alejandra de Racovsky (1924), Nistecha Projer, Demetrio Petrenco (1925), Lelia Anita Cayrus, Alda Esther Cayrus, Sofía Racovsky, Elsa Dreher (1928), Miguel Gordienko, Matilde C. de Gordienko, Paulina C. de Clemenco, Basilio Clemenco, Juan Clemenco, Zoila P. de Clemenco (1931), los Hnos. Stepaniuk (1937), Héctor L. Martigani, Víctor Kosiak, Basilio Kosiak, Catalina Kosiak (1940), Atanacia T. Sandes, Dominga M. Dutra, Leoncio Dutra, Camila Dutra, Irma Dutra, María Lourdes Hornos, Félix Sastre, Pedro Tatarchenco, Leoncio Perfecto, Basilio Oleynick, Adela E. Oleynick, Omar Oleynick, José Oleynick, Juan Kosiak, Melania de Kosiak (1941), Roberto Echeveste, María E. Cayrus, Clara A. Acuña, Demetrio Estarchenco (1942), Francisca de Ayala, María Ayala, Vicenta Argentina Rodríguez, Micaela La Rosa, Carolina Pacheco, Juana de Cánepa, Humberto Cánepa, Esmeralda Cánepa, Edilia B. de Vidal, Rubén E. Cayrus, Walter Plenc, Gladys Plenc (1943), Claudio Ayala, Ramona de Harmas, José Blanco, Carlos Cayrus, Luis. E. Cayrus (1945), Cándida de Curti, Eduardo Cayrus (1948), Juan Kosiak, Ana Kosiak, Pedro Kosiak, Carlos Ayala, Bernardo Cánepa, Humberto Cayrus, Delia Cayrus (1949), Hna. Echeveste (1950), Julia Barboza, Ema Barboza, Roberto Cayrus, Sara Kosiak, Blanca y Silvia [...], René de Cayrus, María de Oleynick, Rosa de Acuña (1951), Julio Godoy, Elida Godoy, Elías Barboza (1953).

${ }^{11}$ Muchos de los inmigrantes rusos que conformaron el primer núcleo de creyentes eran sabatistas, con ciertas dificultades para 
Se dice que en la reunión del primer sábado de enero de 1925 algunos hermanos se sintieron obligados a retirarse "para evitar más disgustos en el día del Señor". Otros simplemente fueron a vivir a otros lugares. ${ }^{12}$ Para 1941 la iglesia contaba con 58 miembros en lista.

Los oficiales de la iglesia se renovaban anualmente. En los primeros 30 años la iglesia contó con los siguientes oficiales:

Ancianos: Miguel Dreher, David Plenc, Héctor Peverini, Basilio Tatarchenco, Pedro Cayrus, Günther Geisse, Jesús Godoy, Pablo Enrique Cayrus.

Diáconos: Eumen Racovsky, David Plenc, Juan Emilio Cayrus, Pedro Cayrus, Pablo Enrique Cayrus, Basilio Tatarchenco, Miguel Dreher, Juan Apoteloz, Jesús Godoy, Juan Kosiak, Héctor L. Martigani, Carlos Dreher.

Diaconisas: Constancia Davit de Cayrus, Cristina Z. de Dreher, Emilia Dreher de Cayrus, Ana S. de Cayrus, María D. de Godoy, Catalina D. de Godoy, Estela D. de Cayrus, Margarita Cayrus de Garbarino, Malagia Kosiak.

adaptarse a todas las doctrinas adventistas. Por diferencias doctrinales o por apostasía, dejan la iglesia en esos años Procopio Nischeta, Demetrio Petrenko, Basilio Clemenco, Juan Clemenco, Paulina Clemenco, Ivila de Clemenco, Basilio Tatarchenco, Demetrio Tatarchenco, José R. Blanco, Camila Dutra, Irma Dutra, Esmeralda Cánepa, Clara Acuña, Lioncio Dutra y Basilio Oleynick.

${ }^{12}$ Así ocurrió con Carlos y Juan Racovsky, Miguel y Matilde Gordienko, David y Elena Plenc. Benoní Cayrus y Elvira Oleynick se trasladan a Puiggari y se unen a la iglesia en ese lugar. Otros que se mudaron fueron Elena de Tatarchenco, Rita Cayrus de Caballero, Elvira Kosiak, María Ayala y María Ester Cayrus. Los docentes de la escuela también se alejaban luego de haber cumplido su labor por algún tiempo. 
Secretarios de iglesia: Juan Emilio Cayrus, Alina Cayrus, Emilia Dreher de Cayrus, David Plenc, Paulina Cayrus (de Barboza), Estela Dreher de Cayrus, Pablo Enrique Cayrus, Inés Cayrus (de Martigani), Esli Eber Cayrus, Olga Dreher, Günther Geisse, Jesús Godoy.

Tesoreros: Carlos Racovsky, Paulina Cayrus (de Barboza), Miguel Dreher, Pedro Cayrus, Juan Emilio Cayrus, Pablo Enrique Cayrus.

Directores misioneros: Miguel Dreher, David Plenc, Pedro Cayrus, Basilio Tatarchenco, Carlos Dreher, Jesús Godoy, Juan Emilio Cayrus, Josefa Ramos.

Secretarios misioneros: Elena Cayrus de Plenc, Margarita Cayrus, Luisa Dreher, Olga Dreher, Esli Eber Cayrus, Carlos Dreher.

Directores de jóvenes: David Plenc, Pedro Cayrus, Pablo Enrique Cayrus, Benoní Cayrus, Esli Eber Cayrus, Alda Cayrus de Geisse, Carlos Dreher, Olga Dreher, Héctor L. Martigani, Ataídes Luz, Josefa Ramos, José Oleynick, Yolanda Kalbermatter, Inés Cayrus de Martigani.

Directores de Escuela Sabática: David Plenc, Carlos Racovsky, Pedro Cayrus, Pablo Enrique Cayrus, Günther Geisse, Carlos Dreher, Miguel Dreher, Esli Eber Cayrus, Basilio Oleynick, Jesús Godoy, Héctor L. Martigani.

Directoras de la Sociedad Dorcas: Sinforosa G. de Apoteloz, Paulina Cayrus de Barboza, Alda Cayrus de Geisse, Margarita Cayrus de Garbarino, Ana S. de Cayrus.

Nuevas actividades religiosas se añadieron con el transcurso del tiempo. El pastor Sherman incentivó en 1926 el surgimiento de las reuniones de oración los 
días miércoles. En 1945 se realiza una ceremonia de investidura de las Clases Progresivas en la cual varios jóvenes reciben sus distintivos de Amigos y Guías Mayores. También eran evidentes las limitaciones, a veces severas. En más de una oportunidad las "reuniones de negocios" lucharon con problemas económicos y necesidades insatisfechas.

El proyecto de la construcción de la capilla de Colonia Pintos Viana fue muy significativo y desafiante. En 1936 se inicia la recolección de fondos y se nombra una comisión para la búsqueda de un terreno. El acta de la construcción de la capilla dice que los planes concretos se trazaron a fines de 1939 y que Günther Geisse y su esposa Alda Cayrus donaron el terreno. Los Hnos. Cayrus donaron ladrillos, la Misión dio algunos materiales usados y el Pr. Brouchy trabajó como carpintero para arreglar puertas y ventanas. Hermanos y vecinos realizaron otras donaciones. La capilla cuenta con una nave de seis por diez metros y una pieza contigua de tres y medio por seis metros. La inauguración ocurrió el 23 de marzo de 1940, con la presencia del pastor Pedro M. Brouchy y hermanos de Paysandú. El humilde recinto reemplazó a las casas de familia para las reuniones de la iglesia.

Una decisión fundamental fue el establecimiento de una escuela de iglesia. El pastor Mario Rasi dirige las deliberaciones en setiembre de 1944. El estudio previo mostraba la existencia en el área de unos quince niños en edad escolar, aunque llegó a tener más de veinte en los años que siguieron. La pieza contigua sirvió como sala de clases para la Escuela Primaria Adventista "José Pedro Varela" que funcionaba en dos turnos, con un solo docente. Juan Emilio Cayrus donó sillas y Paulina 
Cayrus prestó una mesa. Algunas mejoras debían hacerse, como un aljibe y dos servicios sanitarios. Nuevas sillas y mesas se trajeron de Paysandú. El mobiliario que hoy puede observarse no podría ser más austero. Un pizarrón, siete bancos, una pequeña biblioteca y un par de armarios. Pero los recursos humanos, colocados en las manos de Dios, hicieron la diferencia. La Junta de la Iglesia pidió a la Misión Uruguaya el envío de un maestro, y para febrero de 1945 ya estaba presente el hermano Ataídes Luz. Miembros y vecinos comenzaron a enviar a sus niños a la escuela de la iglesia. ${ }^{13}$ Josefa Ramos se incorpora a la escuela en 1947. En 1950 llega Yolanda Kalbermatter. Los antiguos hermanos de la zona tienen memoria de aquellos maestros cristianos, a los que se sumaron posteriormente Armando Morais, Delia Cayrus de Gerber, Edwin Mayer y María de los Ángeles de Cayrus. ${ }^{14}$

En la flamante capilla se reunían los colonos creyentes para adorar a Dios y de tanto en tanto para ser testigos del surgimiento de nuevos hogares cristianos. El 15 de octubre de 1941 se pide la bendición de Dios sobre el matrimonio de Inés Cayrus y Héctor L. Martigani. El 11 de julio de 1945, unen sus vidas Rita Inés Cayrus y Jaime A. Caballero, con la bendición del

${ }^{13}$ Juan Emilio Cayrus envía tres niños, Pablo Enrique Cayrus dos, Pedro Cayrus uno, Paulina Cayrus de Barboza dos, Silvio Garbarino uno y Miguel Dreher uno. Otras familias que no pertenecían a la iglesia mandaron a seis niños en los inicios de la escuela. A comienzos del siguiente año se inscriben 19 alumnos.

${ }^{14}$ María de los Ángeles de Cayrus enseñó en dos oportunidades, en los años 1954-1955 y en 1958. 
pastor Mario Rasi y la presentación de un canto especial por parte de Ataídes Luz y Olga Dreher. El 9 de enero de 1946, el pastor Rasi oficia en la boda de Olga Dreher y Ricardo Benech, de Nueva Helvecia. El 2 de enero de 1952, el pastor José Tabuenca conduce la ceremonia de casamiento de María Ester Cayrus y Ataídes Luz.

\section{El desafío de crecer y perdurar}

El pueblo de Guichón fue desde el comienzo un importante objetivo misionero. En 1936 se habla de una campaña de evangelización en esa localidad. En 1941, se informa de la presencia de hermanos e interesados en la "Colonia Juncal" y se planifican conferencias evangelizadoras breves en ese lugar. Los oficiales de iglesia deben repartirse entre la Colonia y el pueblo de Guichón. En los años posteriores se concretan diversos planes de visitación de estos hermanos.

En mayo de 1943 se celebra un congreso regional en la iglesia de Guichón con la presencia de los pastores EnriqueWestphal, Mario Rasi, MarceloPidouxy Santiago Mangold, los que posteriormente llevaron a cabo una serie de conferencias evangelizadoras en el pueblo. Los hermanos e interesados se reúnen en un local alquilado y posteriormente en casa de Juan Emilio Cayrus. En el año 1945 se habla del grupo de Guichón y Juncal, bajo la dirección del hermano Pedro Cayrus. En las elecciones de 1947 se designan oficiales para la Colonia Juncal. ${ }^{15}$

${ }^{15}$ Anciano: Pedro Cayrus, diácono: Günther Geisse, tesorero y director de Escuela Sabática: Juan Emilio Cayrus, secretaria de grupo: María Ayala, directora de jóvenes: María E. Cayrus, subdirector de jóvenes: Rubén E. Cayrus. 
A fines de 1952 los hermanos del campo solicitan a la Misión Uruguaya que se haga cargo de los miembros de Guichón como una congregación independiente. Para 1953 el hermano Juan Emilio Cayrus era el director del grupo que se reunía en su casa.

Muchos de los nombres fundadores de la iglesia en la Colonia Pintos Viana se repiten en los registros a lo largo de los años. Como ha ocurrido con muchas áreas rurales, la iglesia allí ha sufrido la emigración de parte de sus miembros. Personas que dieron sus primeros pasos en la iglesia y la escuela de Guichón se trasladaron a diversos lugares en busca de nuevas oportunidades de estudio, trabajo y servicio. Cuando se tomó asistencia en la Escuela Sabática del sábado 27 de enero de 2007, los apellidos mencionados fueron Cayrus, Garbarino, Kirichenko, Kosiak, Presentado, Pereyra y Vidal.

La iglesia de Colonia Pintos Viana sigue existiendo, consciente de sus raíces y de su misión de preparar misioneros y obreros laicos capaces de ofrecer al mundo un mensaje de esperanza. En 2004 se celebraron los 80 años de su fundación, momento propicio para recordar que la fe bíblica perdura y da frutos de vida eterna en los corazones sinceros.

Recorro una vez más el pequeño recinto vacío del templo. Los ecos del hermoso culto del día anterior se han apagado. Miro los pizarrones, los pupitres y los libros que ya no se usan para la escuela de iglesia. Observo otra vez a aquellos hombres, mujeres y niños de las fotografías, excesivamente formales para ese ambiente rural y renuevo mi decisión de permanecer en las filas de aquellos que con entrega y autenticidad iniciaron un camino de fidelidad y compromiso con la misión. : : 